answered. For example, an operation is performed for the removal of some hindrance to the strength or activity of a limb. As soon as the patient has recovered from the operation the case is published, or at all events from that date no further information is given or perhaps obtained of the result ; yet clearly the chief fact which requires to be ascertained is the condition of the limb at a remote period. If the operation be really successful, by the omission of such knowledge an injustice is done to it. If it be not successful, then by such silence an injustice is done to surgery.

Again, I cannot help thinking that the practice is, in some instances, too common of throwing the responsibility of an operation upon the patient. Surgeons perhaps are sometimes too prone, as the result of deliberation, to declare, Well, I will put the matter before the patient and leave him to decide. Now it strikes me that this can hardly ever be quite right. As Sir James Paget has said, "As to the amount of good which is to follow an operation the surgeon alone can in most cases estimate it. In most cases therefore we must take the whole responsibility of operation, for it is only on our statements that patients can rely in judging whether they should submit or not; and most of them, even when they have our statements before them, are quite incapable of clearly and soundly judging." And not only so, but too often it is in proportion to the degree of doubt in the surgeon's own mind that he becomes anxious to shift the responsibility. When the case is clear to him he expresses a positive opinion, but when the advantages and objections are more equally balanced he attempts to place them before the patient and to leave the issue to him. It is therefore in cases in which decision is most difficult that the patient is called on to decide. This ought not to be. Sir James Paget would make an exception in cases of what he wittily calls "decorative surgery." But I confess it seems to me that even this exception should be admitted only with considerable qualification. For when a man or woman is promised a better appearance as the result of an operation, how can either judge of its degree? When, for example, a surgeon proposes to make a new nose, can the patient picture at all adequately the new feature? And yet this is a point of the highest importance in coming to a decision between a new organ of flesh and an artificial one. I think that whenever the surgeon places the whole case fully and fairly before the patient he should at least never withhold his own opinion and the instances ought surely to be very exceptional indeed in which he should act in opposition to it.

\section{STEWED FRUII FOR THE GOUTY AND THE DYSPEPTIC.}

BY J. MILNER FOTHERGILL, M.D.,

PHYSICIAN TO THE CITY OF LONDON HOSPITAL FOR DISEASES OF THE CHEST, VICTORIA PARI.

Probaly the impression first created by scanning the title of this paper will be as follows:- "Why, what have either the gouty or the dyspeptic to do with stewed fruit at all?" That sugar is apt to disagree with sundry stomachs, causing great acidity, is a clinical fact not to be disputed. But because such is the case with a limited number of persons it does not seem, to me at least, that therefore a sweeping prohibitory law is to be laid down for a large section of the community. Grout poison, all admit, is a product derived from the albuminous constituents of our food, as nitrogen is a marked element in its composition. It is, then, the albuminous element in our food which has to be avoided in lithiasis. How sugar, fruits, and even vegetables came to be banned my researches have not enabled me to ascertain. From the time of Magendie's path-breaking essay writers on gout have advised the restriction of the nitrogenised, or azotised, constituents of our food in cases of lithiasis. But that the objection to sugar in gouty cases exists may not be denied. In speaking of stewed fruit for the gouty and the dyspeptic my views will be heterodox in the eyes of many. But neirher the light of chemistry nor the lessons of practice conflict with my views. I am quite prepared to undergo any criticism these views may elicit or provoke. Indeed, the subject would be all the better for being thoroughly ventilated.
At the outset, I admit that for many persons-gouty, dyspeptic, and glycosuric-ordinary stewed fruit is objectionable from the amount of added sugar it contains. Where the acidity of fruit is masked or hidden by an excess of sugar, then the resultant product is cloying to many palates, and offensive to many stomachs. Probably in this all readers will agree with me. But it is by no means necessary to render stewed fruit objectionable by adding much sugar to it. Deprived of this excess of added sugar, stewed fruit can not only be rendered unobjectionable, but be converted into an actual prophylactic measure, especially in cases of lithiasis. In order to attain this end all that need be done is to neutralise the excessive acidity by an alkali, and then little or no sugar is required. Thrifty housewives have long been familiar with the fact that the addition of a small quantity of the bicarbonate of soda to stewed fruit reduced the acidity, so as to save the necessity for much sugar. This was done simply for economy. The principle has a far wider application. Last June I was requested to visit a lunatic in the Midlands who was also gouty; and when the gout was acutely present she was more excitable and violent than usual. No medicine would she take, but she was very fond of stewed fruit. To add potash to her stewed fruit was very easy, yet very effectual. After this I gave my cook instructions to perform a series of experiments for me with all our ordinary native fruits. The result of this was that the amount of bicarbonate of potash required for each pound of fruit was found to be about as much as would lie upon a shilling. And this is a much better guide for a cook than to put so many grains. With all fairly ripe fruit this was just sufficient to neutralise the acidity, and bring out the natural sweetness; indeed the resultant product was quite sweet enough for most adult palates. Such stewed fruit could be eaten alone, or with milk puddings, or with cream, or the Swiss milk in bottles. Gooseberries, currants of all kinds, apples, and plums, all alike were excellent when so prepared. There are some points, however, to be attended to in practice, which are of more or less importance. The first matter is this : with dark fruits, as the black plum, for instance, the colour is impaired by the alkali, and the fruit is less attractive to the eye than is that of the ordinary stewed fruit, which is of a deep clear crimson. This matter is easily got over : a little cochineal will give the desired colour. Another is this: where there is no natural sweetness to neutralise the acid completely by an alkali leaves nothing, simply a cold mass, to which the palate is absolutely indifferent. Such is the case with rhubarb. Here it is well to use half or all the amount of alkali with some sugar. The same is the case with early gooseberries before they have any natural sweetness; no sugar formed in them. Here the full quantity of alkali should be used, and the remaining acidity met by sugar. Where three-quarters of a pound of sugar is required to sweeten one pound of fruit, only one-quarter of a pound of sugar is necessary after the alkali has been added. The sour-sweet taste is thus secured, which is toothsome. Now, in these two instances the stewed fruit is only rendered less objectionable to the stomach plagued with acidity, not made quite inoffensive. But for ordinary gouty individuals not troubled with acidity of the stomach, such stewed fruit is quite admissible, and forms a pleasant method of taking potash. The whole subject is one which deserves attention from invalids as well as their medical attendants, as it opens up to many a new field of diet altogether. Fruits au naturel-as the strawberry, for instanceare good in gout from the salts they contain, and are unobjectionable stewed, if it were not for the acetous fermentation of the added sugar. Here soda may be used. But where there is lithiasis the alkali ought to be potash. The gouty and the bilious alike are troubled with the products of the metamorphosis of albuminoids. Neither the lithates of the gouty nor the bile acids of the bilious are derived from the saccharine or farinaceous elements of the food. It was possible to make bricks without straw, but it is impossible to make bricks without clay! No liver can make these nitrogenised substances from simple hydrocarbons: it is physically impossible! The dietary for each is the same - a non-nitrogenised dietary, in which vegetables and farinaceous matters are indicated, and saccharine matters, too, unless acidity in the stomach is produced by them. Milk puddings and stewed fruit are excel. lent for the dyspeptic, the bilious, and the gouty, as my. experience tells me ; and for one of those who suffers from taking sugar nineteen would be all the better for stewed 
fruit. But for those who dislike sweets, and for those who suffer from acidity, it is well to prepare the stewed fruit with alkalies, completely and solely or partially; as the case may be. This may sound very heterodox to some readers; but $\mathrm{ju}-\mathrm{t}$ let the incredulous test the matter for themselves.

Now, there are two other matters remaining to be alluded tn, on whith it is impossible to speak dogmatically or ex cathedro ; they are, rather, matters of personal belief, and they are these:- 1 . It does not seem a matter of indifference in lithiasis what forms of albuminoids are taken. The flesh of animals is rather converted into peptones by pepsin in an acid medium - that is, by gastric digestion-than by trypsin in an alkaline medium. Aud such peptones seem speciallo liable to form lithates. Caseine is more specially digested by trypsin in the intestine, and such caseine peptones seem less readily converted into lithates; the clinical fact being that a milk dietary or a pulse dietary is good for the subjects of lithiasis. Caseine is the form of albuminoid it seems to me, best suited to the gouty. Milk or milk puddings (made without eggs) are capital food for the choIfemic or the lithæmic. These fibrin-albuminoids, digested hy pepsin, are laxative, while caseine is binding or con. stipating.

Milk puddings, then, go well with stewed fruit, which is a laxative. Many thoughtful physicians agree with me in the above matter. Now I am approaching what some may hold very disputable ground, yet nevertheless I venture to say here what I am beginning to think. Both for the classical diabetic and the glycosuric, cane sugar-the sugar of commerce-is bad, producing the unpleasant symptoms of sugar in the blood very readily. Yet many glycosuric individuals can take farinaceous matter with comparative immunity from discomfort. Starch in its way to grape-sugar is much less troublesome than is cane-sugar passing into grape-sugar, why I do not know, but the fact remains. Now, with many glycosuric individuals fruit stewed in the manner advocated here is quite permissible, while ordinary stewed fruit is very objectionable. For the dyspeptic, the gouty, the bilious, and the glycosuric individual (as well as the truly rheumatic, a small class), fruit stewed with an alkali in the proportion of as much bicarbonate of soda as will lie upon a shilling to the pound of fruit, when put in the oven will be found both palatable and permissible. It saves the gastric acidity from the acetous fermentation of the sugar in the dyspeptic, or with the glycosuric relieves him from the excess of cane-sugar which disagrees with him. Where there is distinct gout not only is fruit stewed with an alkali good and unobjectionable; but if it be prepared with the bicarbonate of potash, it is converted into a therapeutic agent of no mean value; while the resultant product is quite sweet enough for a palate which has outlived the "sweet-tooth" period. The whole matter is a simple one, yet it seems to contain much promise for many persons.

Park-street, W.

\section{ON RUPTURE OF THE BLADDER. WITH NOTES OF TWO CASES.}

By HENRY MORRIS, M.A., F.R.C.S. ENG., SURGEON TO THE MIDDLESEX HOSPITAL.

IN the very able articles by Mr. Rivington on "Rupture of the Urinary Bladder," published in THE LANCET during last year, the author stated at the outset that one of his objects was "to examine critically the cases which have been adduced as instances of recovery after rupture into the peritoneal cavity." In a later number ${ }^{2}$ he discredits the diagnosis in seven out of the eight recorded cases of recovery which he had collected, and amongst them is one reported by me.

Though Mr. Rivington has included my case in the intra. peritoneal group, with the result of turning it out again, I wrote of it: "There is no doubt the wound was in the front wall of the bladder, chiefly, though perhaps not entirely, extra-peritoneal, and being high up in the organ, comparatively little urine escaped through the rent, while the bladder preserved some of its power of retaining and expelling its contents." Mr. Rivington takes exception to the diagnosis on the ground that " the evidence of distension of the bladder

1 The Lancet, June 3rd. I Ibid., Nov. 4 th.

1 The Lancet, June 3rd. I Ibid., Nov. 4 th. is defective, as the man had passed water about an hour before the injury. According to the patient himself, he passed by his own efforts three-quarters of a pint of urine in the first thirty-six hours, and very likely the quantity was greater. ..... Plenty of urine was drawn off daily through an elastic catheter fixed in the bladder." The uncertainty as to whether the peritoneum is torn in any given case of ruptured bladder is so great that it may be plausibly questioned until actually seen, and Mr. Rivington may perhaps be right in his conclusion that the peritoneum in ny case was not ruptured; but his summary of the evidence is a little misleading. My description was: "The patient stated that prior to the injury he had not passed water for ' an hour or two' (to use his own words), and that he had been drinking up to the moment of wrestling. $\mathrm{He}$ also said that during the thirty-six hours between the accident and his admission into the hospital he had 'certainly not passed more than three-quarters of a pint of blood aud uriue together' (again using his own words); that he had made several efforts before he voided anything at all, that which first passed being like pure blood, but that which was passed at subsequent attempts became more and more like natural urine." Mr. Rivington has himself shown that some forms of rupture of the bladder, without fracture of the pelvis, may occur when the organ is empty or almost so. His fourth case is an illustration thereof. But there is sufficient evidence, I think, that in my patient the bladder must have been distended above the level of the pubes, and that urine must have escaped from it at some spot; whilst it supplies just the set of conditions which all consider to be peculiarly predisposing to rupture of the bladder should injury occur to the abdomen, especially if the violence be applied, as it was to my patient, by someone kneeling upon the belly.

Quite apart from its particular reference to my case, the argument that the biadder is not ruptured because thirtysix hours after the injury the patient passes urine by his own efforts, and that subsequently plenty of urine can be daily drawn off, cannot be seriously maintained. Many cases disprove it, but none better than Mr. Rivington's own. Thus, in Case l, some hours after the injury the medical attendant passed a catheter, and drew off a large quantity of blood. Death followed on the fourth day. There was a rent in the upper and posterior part of the bladder, and the recto-vesical pouch was filled with a blood-stained fluid. In Case 2, three hours after the injury five ounces of urine highly coloured with blood were drawn off ; within thirty or thirty-six hours afterwards, sixteen ounces were drawn away, and on the third day ten ounces were drawn off, and six were passed by the patient; on the fifth day he again passed water by himself, "and probably more with a fluid motion." He died on the sixth day. "The rent in the bladder was in the upper and posterior part, and was vertical in direction.......Its lips were glued together by lymph, and it was undergoing repair. The bladder was not completely contracted...... There was a considerable quantity of flaky, turbid, urinous fluid in the pelvis." In Case 3 the house-surgeon drew off eight ounces of blood and urine, and during the four days the patient lived six or eight ounces of sanguineous urine were drawn off night and morning. At the post-mortem "a hole was discovered on the right side at the antero-lateral aspect of the neck of the bladder." In Case 4 there was incontinence of urine after the accident; bloody urine dribbled away, and on the introduction of the catheter more was drawn off, "the urine being expelled with some force. ...... No pain attended either the act of micturition or the passage of the catheter." The bladder was rent just above the prostate gland. In THE LANCET ${ }^{3}$ there is a record of another fatal case in which it is stated that shortly after the accident a catheter was introduced in to the bladder, and urine and clotted blood were drawn off. There was a rupture to the right of the vesicula seminalis, and "a quart of urine and blood-clot were found on the right side of the pelvic cavity."

Other cases showing the same facts might be quoted, but enough has been said to justify this very important induction-that neither the presence of urine within the bladder, nor its withdrawal by catheter, nor even its discharge by the patient himself shortly after the accident, vegatives rupture of the urinary bladder; and, further, that urine may be daily withdrawn through a catheter or voluntarily voided by the patient after either intra- 